\title{
Diseño de un cuestionario para evaluar conocimientos básicos de estadística de estudiantes del último curso de ingeniería
}

\author{
Esperanza Ayuga-Téllez, Concepción González-García, María. A. Grande-Ortiz y \\ Eugenio Martínez-Falero \\ Grupo de Innovación Educativa en Técnicas Cuantitativas Aplicadas a la Ingeniería \\ Medioambiental. Universidad Politécnica de Madrid. ETSI Montes. Ciudad Universitaria s/n, 28040 \\ Madrid-España. (e-mail: esperanza.ayuga@upm.es; m.angeles.grande@upm.es)
}

Recibido Nov. 08, 2011; Aceptado Dic. 13, 2011; Versión final recibida Ene. 19, 2012

\begin{abstract}
Resumen
Se presenta el diseño de un cuestionario para evaluar conocimientos básicos de estadística para estudiantes de ingeniería. El cuestionario original incluyó un amplio número de ítems agrupados por bloques temáticos. Este se propuso a un panel de expertos, formado por nueve profesores de la asignatura de Estadística Aplicada de distintas titulaciones de ingeniería en universidades iberoamericanas. Este grupo de expertos evaluó las preguntas planteadas y entregó información valiosa para preparar el cuestionario definitivo. El cuestionario que se propone incluye 20 preguntas repartidas en cuatro bloques temáticos: estadística descriptiva, técnicas de muestreo, pruebas de hipótesis y modelo lineal general. Estos bloques temáticos son consistentes con los resultados de estudios anteriores sobre valoración de conocimientos estadísticos.
\end{abstract}

Palabras clave: evaluación del aprendizaje, ingeniería, estadística, panel de expertos

\section{Design of a Questionnaire to Assess Knowledge of Basic Statistics of Senior Engineering Students}

\begin{abstract}
The design of a questionnaire to assess knowledge of basic statistics for engineering students is presented. The original questionnaire included a large number of items grouped by subjects. This questionnaire was submitted to a panel of experts, consisting of nine teachers of Applied Statistics from different engineering majors belonging to iberoamerican universities. This group of experts evaluated the questions and provided valuable information to prepare the final questionnaire. The final questionnaire proposed includes 20 questions divided in four subjects: descriptive statistics, sampling, hypothesis test and general linear model. These subjects are similar to other studies found in the literature on evaluation of knowledge of basic statistical concepts.
\end{abstract}

Keywords: learning evaluation, engineering, statistics, panel of experts 


\section{INTRODUCCIÓN}

Los procesos de medición y evaluación buscan especialmente identificar los efectos, los impactos y la eficiencia de la inversión en el desarrollo de proyectos o actividades específicas (Cardona y Sánchez, 2010). En los últimos años se ha observado un interés creciente por la evaluación del aprendizaje a gran escala, lo que ha permitido un mejor entendimiento y caracterización del logro educativo de los estudiantes (Maher, 2004; Martínez-Padilla y Pérez-González, 2008; Cancela et al., 2010). La adaptación de los estudios universitarios al Espacio Europeo de Educación Superior (EEES) y la incorporación de los procesos de acreditación de titulaciones e instituciones en Europa, hace necesario replantear los conocimientos, así como las capacidades y destrezas que con ellos deben adquirir los estudiantes universitarios. La evaluación de la eficacia educativa proporciona información de gran importancia tanto para las Instituciones de Educación Superior como para la elaboración de programas y cursos concretos (Nowatzki, 2004; Bornmann et al., 2006). Sin embargo, la selección de indicadores apropiados de dicha eficacia es una tarea difícil, especialmente cuando los criterios no están bien definidos (Praslova, 2010).

La evaluación de los conocimientos de estadística y su comprensión se han desarrollado en algunos trabajos para diferentes niveles educativos (Gardner y Hudson, 1999; delMas et al., 2007; Díaz y Batanero, 2009; Vendramini et al., 2010). Por otra parte, los trabajos publicados relacionados con evaluación de la eficacia educativa en la enseñanza superior son escasos y la mayoría a nivel Nacional y con una temática muy concreta (p.e. Downs, 2006, Damian et al., 2010). En la actualidad se está desarrollando en Europa el proyecto AHELO (OECD, 2010) que consiste en evaluar mediante un test el desempeño de los graduados en dos disciplinas: Economía e Ingeniería. La evaluación de los resultados del aprendizaje se desarrolla mediante diversas pruebas. Algunas de ellas son aplicaciones de cuestionarios sobre conocimientos básicos de cada disciplina (GNE, 2011).

En concreto, a los alumnos de una universidad específica en titulaciones de ingeniería, como es la Universidad Politécnica de Madrid (UPM), se les presupone unos conocimientos en materias básicas que es preciso verificar con el objeto de planificar los contenidos de los programas en los nuevos planes de estudios. Por esto, son de interés los estudios para establecer los conocimientos mínimos comunes en materias básicas que se deben adquirir para el desarrollo de las actividades propias en ingeniería y arquitectura (Ayuga et al., 2010a; GNE, 2011).

La materia de Estadística tiene especial importancia por sus aplicaciones en ingeniería (análisis de datos, diseño de experimentos, control de procesos, procesos estocásticos, fiabilidad de sistemas,...). Los conocimientos, en Estadística, que debe poseer un egresado en su ejercicio profesional dependerán del trabajo que tenga que desempeñar y siempre aparece un curso de esta materia junto con las Matemáticas, la Química o la Física. Son muchas las referencias que se pueden encontrar sobre su importancia en los programas de ingeniería: a nivel de escuelas técnicas en España (Peña et al., 1990); como consecuencia de los procesos de acreditación, se encuentra recogida la materia de "Probabilidad y Estadística" en el estudio de la Accreditation Board for Engineering and Technology (ABET) de Kohen, 2001, sobre evaluación y revisión de programas de ingeniería civil. Entre las competencias de los ingenieros se encuentran "la gestión de la incertidumbre" y la "toma de decisiones", que se apoyan en la Estadística (Dym et al., 2005). Los requerimientos específicos de la materia dependen de la rama de ingeniería, algunas referencias son: para ingeniería de telecomunicación (Aparicio, 2000), ingeniería eléctrica (Nagy et al., 2008), ingeniería hidráulica (Schwenk et al., 2009).

Para las nuevas titulaciones de grado en España, el RD 1393/2007 que regula las enseñanzas universitarias oficiales, contempla la Estadística como "materia de formación básica de la ingeniería". Por tanto, el análisis de los contenidos y su aprendizaje por parte de los alumnos de la Universidad Politécnica de Madrid (UPM) adquiere especial relevancia (BOE, 2007). Dada la variedad de titulaciones en esta Universidad, que comprenden todas las ramas de ingeniería, existen temas básicos, que se encuentran en todos los programas de asignaturas de esta materia, junto con otros, más específicos, de especial aplicación en titulaciones como Ingeniería de 
Telecomunicaciones. La Estadística se presenta con un menor peso, como materia optativa, sólo en el programa de Arquitectura (Ayuga et al., 2010b).

En este estudio se ha pretendido diseñar un cuestionario que reúna los temas básicos de Estadística Aplicada y que deberían ser comunes a todos los programas y planes de estudios desarrollados en la enseñanza de ingenierías. El cuestionario debería ser útil para la evaluación de los conocimientos que de esta materia deberían tener los egresados de la UPM y, en general, a los de cualquier Universidad que imparta estudios de este tipo.

\section{MATERIALES Y MÉTODOS}

La metodología empleada en este trabajo consistió en la obtención de un panel de expertos (Quiroga, 2008) que evaluó una prueba de conocimientos de estadística para egresados en ingeniería. Los resultados se aplicaron al diseño de un cuestionario de conocimientos básicos en la materia.

El diseño de cuestionarios a través de paneles de expertos es frecuente en la investigación relativa a la evaluación de conocimientos o actitudes (Venter, 2008; Kessell et al., 2009; Oloruntegbe et al., 2010, Childress, 2011). Dichos paneles están formados por un pequeño número de personas (entre 3 y 15) con amplia experiencia en el campo profesional de que se trate. Dichos expertos deben estar muy cualificados en el área objeto de evaluación, y ser reconocidos y respetados por sus pares. La independencia del experto respecto del programa que va a evaluar es de gran importancia ya que el evaluador nunca puede ser juez y parte en lo evaluado. Las ventajas principales de la aplicación de esta metodología a la evaluación de cuestionarios sobre conocimientos son, fundamentalmente, el profundo conocimiento de los expertos sobre los temas objeto de evaluación. Esto implica una gran credibilidad en las conclusiones y ahorros de tiempo y costes del proceso de evaluación. El principal inconveniente de esta metodología es que los expertos limiten su independencia por empatía con el resto. También existe el peligro de que excedan el campo de sus competencias reconocidas.

El panel de expertos se constituyó con profesores de la asignatura "Estadística Aplicada" en diferentes Universidades y titulaciones de ingeniería. Se consideró la posibilidad de incluir profesionales de la Ingeniería con amplia experiencia laboral para completar el panel, pero resultó imposible acceder un conjunto representativo de éstos, dispuestos a realizar dicha evaluación. Inicialmente se remitió el cuestionario a una amplia muestra aleatoria de profesores que reunían las características requeridas y pertenecientes a universidades distintas entre las que no se incluyó la Universidad Politécnica de Madrid, para evitar el sesgo en las respuestas. Los cuestionarios se enviaron por correo electrónico acompañados de una carta de presentación con los objetivos del estudio, para incentivar la participación, ya que el cuestionario planteado es extenso, aunque no requería mucho tiempo de respuesta (aproximadamente 15 minutos)

El panel de expertos lo constituyeron, finalmente, nueve profesores, de los cuales, sólo dos de ellos pertenecen a la misma universidad, aunque a diferentes titulaciones. Tres de ellos imparten Estadística en ingeniería industrial, dos en ingeniería de telecomunicaciones y el resto en agronomía, forestal, informática y en una titulación de postgrado. No se pudo contar con profesores de Ingenierías del grupo de construcción. De los componentes, tres son profesores de universidades iberoamericanas y el resto de universidades españolas. Además, ocho de los expertos incluidos en el panel están adscritos al área de conocimiento de Estadística e Investigación Operativa y sólo uno pertenece al área de Tecnología. La composición del panel, según sus componentes posean o no el título de doctor, presenta cuatro doctores en Ingeniería, tres en Matemáticas y dos en Estadística (Ayuga et al, 2010a). Se comprobó que todos los integrantes del panel de expertos alcanzaran un coeficiente de competencia mínimo de 0,8 (García Martínez et al., 2011)

El cuestionario enviado a los expertos recogía la mayoría de los bloques temáticos que se imparten en las titulaciones de ingeniería de las universidades españolas, ya sea de forma explícita o, como en el caso de prácticas con software estadístico, dentro de las propias 
cuestiones, con gráficos o resultados de programas. Los ocho bloques temáticos incluidos en el pre-test fueron: Estadística descriptiva, Probabilidad, Muestreo y estimación, Test de hipótesis, Modelo lineal general, Diseño de experimentos, Procesos estocásticos y Análisis Multivariante. Cada bloque incluyó diez cuestiones tipo test, y el panel de expertos debía elegir cuatro de cada bloque. En este caso, los ítems a valorar eran más del doble de los elementos necesarios para que se puedan descartar cuestiones poco importantes o inadecuadas en la valoración previa (Parmenter y Wardle, 2000).

Las cuestiones eran del tipo respuesta múltiple, ya que éstas requieren menos tiempo de aplicación y son fáciles de responder, puntuar y procesar de forma objetiva y fiable, como se recoge en los trabajos de "Gronlund (1993) Make Achievement Tests and Assessments, Nunnally (1972) Educational Measurement and Evaluation y Babbie (1975) The Practice of Social Research" mencionados por Venter (2008). Se escogió construir elementos con tres posibles respuestas y con una sola opción correcta (Shizuka et al., 2006; van de Watering et al., 2008). No se incluyeron en las posibles opciones, alternativas como "ninguna de las anteriores" o "todas las anteriores" que se consideran poco recomendables en este tipo de cuestionarios. Las respuestas se distribuyeron de forma aleatoria, por lo que cada alternativa $(a, b, c)$ estuvo representada de forma equilibrada en cuanto a la corrección de la respuesta (Venter 2008).

En total los expertos debían valorar 80 cuestiones. Algunas de ellas de dificultad baja, sobre tipos de variable, valores centrales, distribución de probabilidades; y cuestiones de mayor dificultad sobre estimación puntual, estimación por intervalos, contrastes de hipótesis, análisis de la varianza, regresión lineal, regresión múltiple o diseño de experimentos.

Las cuestiones relacionadas con la estadística descriptiva fueron del tipo identificación de tipos de variables (ítems 1 y 2), interpretación y cálculo de medidas centrales (ítems del 3 al 8) e interpretación de gráficos (ítems 9 y 10). Las cuestiones sobre Cálculo de Probabilidades incluidas en el cuestionario pedían evaluar probabilidades de sucesos sencillos con y sin influencia de la variabilidad (ítems 1 al 3), probabilidad condicionada (ítem 4), modelos cásicos de distribución (ítems del 5 al 8), interpretación de funciones de densidad y distribución (ítems 9 y 10). En el bloque sobre temas de muestreo y estimación, las preguntas planteadas se refieren al cálculo de tamaños de muestra (ítems 1 y 5), características de diferentes diseños de muestreo (ítems del 2 al 4 e ítem 6), características de estimadores (ítems 7 y 8) e intervalos de confianza (ítems 9 y 10).

Las cuestiones relacionadas con tests de hipótesis abarcaron diferentes aspectos generales de los contrastes, como el estadístico del contraste (ítem 1, 8 y 9), tipos de errores (ítem 2), nivel de significación (ítems 3 y 4), características de los contrastes (ítems del 5 al 7 e ítem 10). Las cuestiones planteadas para el tema del modelo lineal general incluyeron el análisis de varianza (ítem 1), coeficientes de correlación (ítems 2 y 7), utilidad del modelo lineal (ítem 3), selección del modelo (ítems 4 y 5), hipótesis del modelo (ítems 6, 9 y 10) y datos atípicos (ítem 8). En el bloque temático del diseño de experimentos se plantearon cuestiones relacionadas con los conceptos básicos (ítems del 1 al 3, del 8 al 10 e ítem 5), sobre la expresión del modelo y su análisis (ítems 4 y 6).Las cuestiones planteadas en el tema de procesos estocásticos están relacionadas fundamentalmente con los conceptos básicos de éstos (ítems del 1 al 6) y con las medidas de dependencia (ítems del 7 al 10). Y, por último, en el análisis multivariable se incluían cuestiones sobre los datos (ítem 1), utilidad de diferentes técnicas (ítems del 2 al 6 e ítems 8 y 9), resultados de los análisis (ítems 7 y 10).

Los expertos debían valorar bloques de temas y cuestiones concretas sobre dichos temas. El objetivo era conseguir un cuestionario de 20 ítems, como máximo, para que no resultara excesivamente tedioso. La primera opción que se planteó a los expertos fue escoger entre los bloques temáticos, indicando el orden de preferencia, numerando los ocho bloques de mayor a menor importancia para el desarrollo profesional del Ingeniero (1 el más importante, 8 el menos importante). El análisis de estos resultados se basó en el concepto de ordenación preferencial de la toma de decisiones (Martínez-Falero et al., 1995). A continuación los expertos debían señalar, dentro de cada bloque, las cuatro cuestiones que consideraran más adecuadas para evaluar los 
conocimientos de Estadística Aplicada que debería poseer un Ingeniero. Esta respuesta se resumió mediante análisis de frecuencias (Ayuga et al, 2010b).

La validez del cuestionario resultante se evaluó en términos de lógica, claridad y grado de dificultad por dos profesores y dos alumnos de últimos cursos de la UPM, todos ellos familiarizados con la materia analizada. Posteriormente la prueba se validó aplicando el cuestionario a un grupo piloto, de características similares a la población en estudio, formado por 31 alumnos que ya habían cursado la materia de Estadística Aplicada en un programa de la UPM.

\section{RESULTADOS}

Con la información aportada por los nueve expertos se construyó una función de valor aditiva, en la que el valor de cada bloque temático se obtuvo por la suma total del número de orden asignado por los expertos. Los mínimos de la función se corresponden con el orden de importancia; es decir, cuanto menor es el valor mayor es su importancia. Los cuatro bloques con función de valor inferior al resto se seleccionaron para elaborar el cuestionario. En la tabla 1 se recoge el resultado de la función de valor para los ocho bloques temáticos.

Tabla 1: Valores de la función de valor para los 8 bloques temáticos.

\begin{tabular}{|c|c|c|}
\hline Bloques & Función de valor & Orden de importancia \\
\hline Estadística descriptiva & 23 & 1 ○ 2 \\
\hline Probabilidad & 54 & 7 \\
\hline Muestreo y estimación & 38 & 4 \\
\hline Test de hipótesis (TH) & 23 & 1 ○ 2 \\
\hline El modelo lineal general (MLG) & 27 & 3 \\
\hline Diseño de experimentos & 40 & 5 \\
\hline Procesos & 68 & 8 \\
\hline Análisis Multivariante (AM) & 51 & 6 \\
\hline
\end{tabular}

Los bloques de Estadística descriptiva y Test de hipótesis resultaron los de mayor importancia (función de valor igual a 23, valor mínimo e igual en ambos casos, por lo que se asigna a ambos bloques el mismo orden de importancia 1 o 2). Procesos y Probabilidad son los bloques con mayor función de valor, aunque los temas correspondientes a Probabilidad son de los más impartidos en la UPM. En la figura 1 se representa gráficamente la función de valor para el conjunto de bloques.
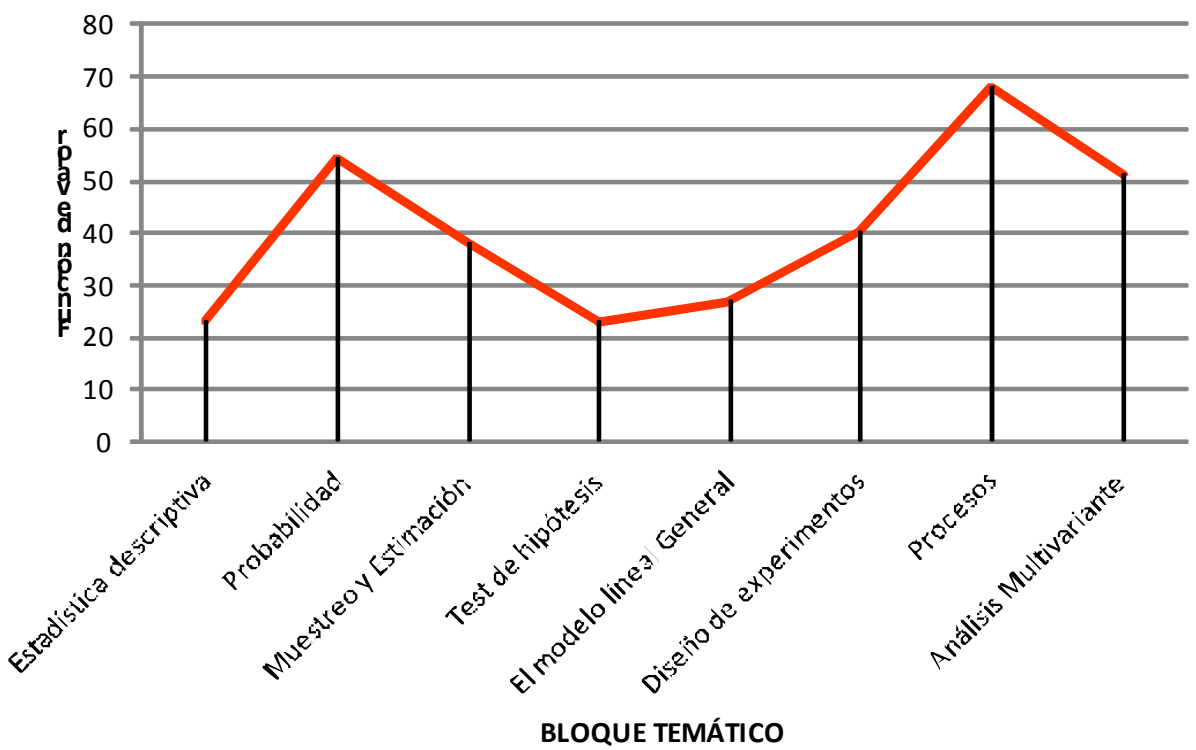

Fig. 1: Función de valor para los bloques temáticos. 
Dentro de cada bloque temático, las cuestiones consideradas más adecuadas para valorar los conocimientos adquiridos por los alumnos se seleccionaron contabilizando el número de expertos que consideraron adecuados dichos ítems. En la Tabla 2 se recogen estos resultados.

Tabla 2: Número de expertos que seleccionaron los ítems.

\begin{tabular}{|c|c|c|c|c|c|c|c|c|c|c|}
\hline Bloques ITEMS & 1 & 2 & 3 & 4 & 5 & 6 & 7 & 8 & 9 & 10 \\
\hline Descriptiva & 3 & 3 & 7 & 0 & 1 & 0 & 6 & 2 & 7 & 7 \\
\hline Probabilidad & 2 & 2 & 1 & 6 & 6 & 3 & 5 & 4 & 6 & 1 \\
\hline Estimación & 5 & 0 & 1 & 6 & 8 & 4 & 3 & 0 & 4 & 5 \\
\hline TH & 5 & 4 & 3 & 5 & 5 & 4 & 5 & 1 & 2 & 2 \\
\hline MLG & 7 & 2 & 5 & 5 & 6 & 4 & 1 & 3 & 0 & 3 \\
\hline Diseño & 6 & 6 & 2 & 3 & 3 & 3 & 2 & 3 & 1 & 7 \\
\hline Procesos & 6 & 3 & 5 & 2 & 3 & 3 & 1 & 2 & 6 & 5 \\
\hline AM & 4 & 6 & 1 & 7 & 0 & 7 & 0 & 9 & 1 & 1 \\
\hline
\end{tabular}

A la vista de estos resultados se determinó incluir cuatro bloques temáticos de 5 ítems por bloque, con un total de 20 cuestiones. Para ello, las profesoras de Estadística participantes en el estudio resolvieron los empates. Los bloques escogidos para la prueba de conocimiento son: i) Estadística Descriptiva; ii) Muestreo y Estimación; iii) Test de Hipótesis; y iv) Modelo Lineal General.

Los ítems relacionados con la estadística descriptiva fueron el 3 , el 7 el 9 y el 10 (con puntuaciones mayores de 5) y entre los ítems 1 y 2, ambos relacionados con la identificación de tipos de variables y elegidos por tres personas del panel de expertos, se seleccionó el 2 . En muestreo y estimación se puntuaron más los ítems 1, 4, 5 y 10, siendo el 6 y el 9 (elegidos ambos por 4 expertos) ítems relacionados con el diseño de muestreo e intervalos de confianza. Se optó por el ítem de diseño de muestreo. En cuanto al tema de test de hipótesis los ítems más valorados fueron 1, 4, 5 y 7. Igual que en el bloque temático anterior, los ítems 2 (error de tipo I) y el 6 (test de la chi-cuadrado) fueron elegidos ambos por 4 expertos; en este caso, se optó por el ítem 6 . En el modelo lineal general, los ítems seleccionados por los expertos fueron el 1, 3, 4, 5 y 6.

La prueba de evaluación que se propone, a raíz de estos resultados, para evaluar los conocimientos estadísticos necesarios para el ejercicio profesional de la Ingeniería se muestra en las tablas 3 a 6 , donde se recogen las cuestiones planteadas para cada bloque temático y las opciones de respuesta. Con la aplicación del análisis del cuestionario al grupo de profesores y alumnos para validar la claridad del mismo no se detectaron inconsistencias en el test. Al analizar los resultados de su aplicación al grupo piloto se detectó cierta confusión en la cuestión sobre el cálculo de la mediana. Entrevistado un grupo de alumnos sobre dicha cuestión, el ítem se modificó en el sentido de presentar de forma más clara los datos para el cálculo, quedando su enunciado tal y como se muestra en la tabla 3.

\section{CONCLUSIONES}

Para la determinación del grado de conocimientos básicos de la materia de Estadística con los que cuenta un egresado de la UPM, se ha elaborado un cuestionario de 20 preguntas repartidas en cuatro bloques temáticos: Estadística descriptiva, Técnicas de Muestreo, Test de hipótesis y Modelo lineal general. Estos bloques temáticos son consistentes con los resultados de estudios anteriores sobre valoración de conocimientos estadísticos Cada bloque incluye cinco preguntas que fueron las seleccionadas por un panel de expertos, a partir de un cuestionario inicial de 80 ítems. Los 20 ítems del cuestionario son del tipo respuesta múltiple con una sola respuesta correcta de tres posibles. El cuestionario se validó, en sus aspectos lógicos y de claridad, con un grupo piloto de más de 30 estudiantes de últimos cursos de la UPM. 
Tabla 3: Cuestiones sobre conocimientos de estadística descriptiva

En un control de calidad se observa el "número de tornillos defectuosos" en cada lote fabricado, ¿qué tipo de variable estadística es?
a) Discreta
b) Continua
c) Cualitativa

En un proceso industrial se mide $X=$ tiempo (en minutos) de espera de llegada de piezas a la envasadora, con los siguientes resultados:

\begin{tabular}{|l|r|r|r|r|r|r|r|r|r|}
\hline Valores $X$ & 99,5 & 99,1 & 53,4 & 23,8 & 9,5 & 3,6 & 2,5 & 0,87 & 0,78 \\
\hline Frecuencia relativa acumulada & 1 & 0,995 & 0,9 & 0,75 & 0,5 & 0,25 & 0,1 & 0,05 & 0,0 \\
\hline
\end{tabular}

El valor de la mediana es:
a) 23,8
b) 16,65
c) 9,5

En una fábrica de motosierras se controla la longitud de la cuchilla y se han obtenido las siguientes mediciones, en centímetros:

\begin{tabular}{|l|c|c|c|c|c|}
\hline Valores $X$ & 58,7 & 60,1 & 61,5 & 62,9 & 64,3 \\
\hline Frecuencia absoluta & 8 & 14 & 8 & 8 & 2 \\
\hline
\end{tabular}

El valor de la media es:
a) 58,95
b) 60,87
c) 62,10

En un estudio se mide la "profundidad de suelo", para representar las frecuencias de los valores de la variable ¿qué tipo de gráfico se emplearía?
a) Diagrama de barras
b) Gráfico de sectores
c) Histograma

En un estudio sobre el "peso de la carga de los camiones" que entran en fábrica se ha obtenido la siguiente figura. ¿Qué representa la línea vertical del interior de la caja?

Box-and-Whisker Plot

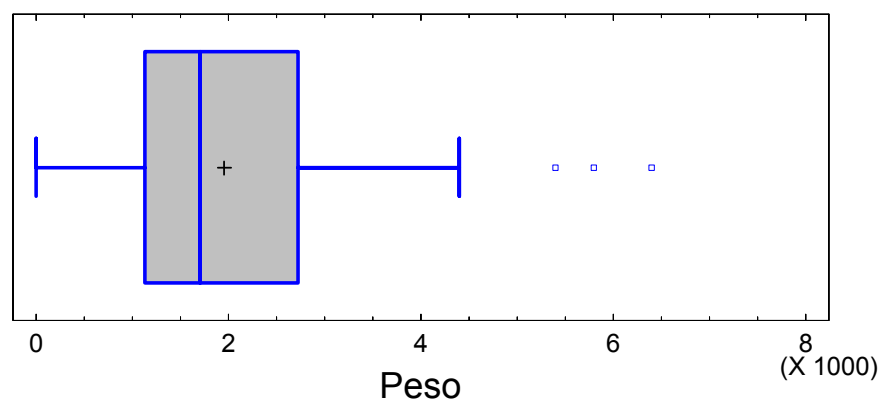
a) La media
b) La mediana
c) La moda 
Tabla 4: Cuestiones relativas a los conocimientos relacionados con los temas de muestreo y estimación

En el cálculo del tamaño " $n$ " muestral, es necesario conocer,

a) El error máximo de muestreo "e" que se está dispuesto a aceptar y el nivel de confianza a para la estimación.

b) El error máximo de muestreo "e" que se está dispuesto a aceptar y la desviación típica de la variable a estudiar o algún valor estimado de la misma.

c) La desviación típica de la variable a estudiar o algún valor estimado de la misma, el error máximo de muestreo "e" que se está dispuesto a aceptar y el nivel de confianza a para la estimación.

Sabemos que la tensión arterial de la población aumenta a partir de los 60 años. Para estimar la "presión arterial media" de los individuos de una gran ciudad, se debe elegir un diseño de muestreo, ¿cuál?:

a) Aleatorio simple

b) Estratificado, con diferentes estratos según la edad.

c) Por conglomerados, con conglomerados según el barrio.

En un muestreo aleatorio simple se desea obtener el tamaño de muestra adecuado para estimar la "proporción de vidrio por kilo de basura reciclable". La expresión que se puede utilizar es:

$$
n_{O}=\left(\frac{1,96}{0,005}\right)^{2} 0,5 \cdot 0,5=38416 \text { kilos }
$$

a) Con un $95 \%$ de confianza, error de estimación $0,5 \%$ y máxima varianza.

b) Con un $95 \%$ de confianza, error de estimación 0,005 y mínima varianza

c) Con un $90 \%$ de confianza error de estimación 0,5 y varianza 0,005

Para realizar un muestreo sistemático en un terreno:

a) Se eligen $n$ puntos de muestreo al azar sobre el total de la superficie.

b) Se superpone una malla con $k \cdot n$ nodos y se escogen al azar $n$ de ellos, de tal forma que, cada uno de los $\mathrm{k} \cdot \mathrm{n}$ nodos tenga la misma probabilidad de ser escogido.

c) Se superpone una malla con $n$ nodos y se escoge al azar un punto de la superficie, superponiendo en ese punto un nodo de la malla. Los n nodos así colocados serán los puntos de la muestra.

Se tienen muestras de diámetros medios de dos especies de pinos medidos a diferentes edades: 5 , $10, \ldots, 150$ años. ¿Qué conviene utilizar para calcular un Intervalo de Confianza para la diferencia de diámetros entre las dos especies?

a) Un intervalo para diferencia de medias con muestras pareadas

b) Un intervalo para diferencia de medias con muestras independientes

c) Un intervalo para cada media diamétrica 
Tabla 5: Cuestiones relacionadas con tests de hipótesis

Para aplicar un contraste de hipótesis, el estadístico del contraste debe medir:

a) La diferencia entre la $\mathrm{H}_{0}$ y la $\mathrm{H}_{1}$

b) La diferencia entre la muestra y la $\mathrm{H}_{0}$

c) La diferencia entre la muestra y la $\mathrm{H}_{1}$

El error de tipo I se comete cuando:

a) $\mathrm{La} \mathrm{H}_{0}$ es verdadera

b) La hipótesis alternativa es verdadera

c) $\mathrm{La} \mathrm{H}_{0}$ es menos probable

El nivel de significación del contraste es igual:

a) Al p-valor (nivel crítico)

b) A la probabilidad de cometer un error de tipo I

c) A la probabilidad de cometer un error de tipo II

Si dos variables cualesquiera siguen una distribución normal, el coeficiente adecuado para detectar su independencia es:

a) El de la $\mathrm{T}$ de Kendall

b) El de Spearman

c) El de correlación de Pearson

Un contraste de bondad de ajuste con la $x^{2}$ debe cumplir que:

a) Las frecuencias observadas deben ser mayores que 5

b) Las frecuencias esperadas deben ser menores que 5

c) Las frecuencias esperadas deben ser mayores que 5

Tabla 6: Cuestiones relacionadas con el modelo lineal general

¿Cuál es la hipótesis nula empleada en el análisis de varianza?

a) La igualdad de varianzas entre grupos

b) La igualdad entre todas las medias de los grupos

c) La veracidad del modelo

Un modelo lineal simple sirve para:

a) Minimizar la dispersión relativa de la variable dependiente.

b) Modelizar relaciones y predecir valores de alguna de las variables relacionadas.

c) Encontrar la relación causa-efecto entre dos variables

Tenemos dos modelos linealizables que pueden utilizarse para predecir el valor de Y. El modelo 1 tiene una $R^{2}=0,87$ y muestra heterocedasticidad residual. El modelo 2 tiene una $R^{2}=0,67$ pero los residuos cumplen todos los requisitos. Decido:

a) Utilizar la recta de regresión, ya que ambos modelos son malos.

b) Elegir el modelo 1 que tiene mayor $\mathrm{R}^{2}$.

c) Elegir el modelo 2 que cumple todas las hipótesis.

En un modelo de regresión se obtiene el siguiente análisis de varianza:

\begin{tabular}{|c|r|r|r|r|r|}
\hline Source & Sum of squares & Df & Mean Square & F-Ratio & P-Value \\
\hline Model & 678,745 & 1 & 678,745 & & \\
Residual & 118709 & 363 & 327,023 & 2,08 & 0,1505 \\
\hline Lack-of-Fit & 6952,76 & 29 & 239,751 & & \\
Pure Error & 111756 & 334 & 334,6 & 0,72 & 0,8605 \\
\hline Total (Corr.) & 119388 & & & & \\
\hline
\end{tabular}

Estos resultados se pueden interpretar de la siguiente forma:

a) El modelo es malo ya que los p-valores del ANOVA son mayores de 0,05.

b) El modelo explica poca variabilidad pero la relación entre $x$ e y es lineal.

c) El modelo explica mucha de la variabilidad pero la relación entre $x$ e y no es lineal.

Los residuos del modelo deben ser: (valores de una variable aleatoria, V.a.)

a) V.a. con distribución $\mathrm{N}(\mu, 1)$; con " $\mu$ = valor constante" e incorrelados entre sí

b) V.a. con distribución $N(0, \sigma)$ con " $\sigma=$ valor constante" e incorrelados entre sí

c) V.a. con distribución $\mathrm{N}(\mu, \sigma)$ con " $\sigma=$ valor constante" y media diferente 


\section{AGRADECIMIENTOS}

Agradecemos la financiación y el apoyo para la realización de este estudio a la Agencia de Calidad, Acreditación y Prospectiva de las Universidades de Madrid (ACAP).

\section{REFERENCIAS}

Aparicio, F.M. Pautas para la Mejora de la Calidad en la Enseñanza de Estadística en Ingeniería de Telecomunicación. Revista ELectrónica de Investigación y EValuación Educativa, ISSN 11344032 (en línea), 6(1) 2000. http://www.uv.es/RELIEVE/v6n1/RELIEVEv6n1_2.htm. Acceso: 17 de Enero (2012)

Ayuga-Téllez, E.; González-García, C.; Grande-Ortíz, M.A.; Reyes-Hernández, B. y GarcíaVentura, C., Statistical topics in UPM engineering degrees. Actas del Joint International IGIP-SEFI 2010, Trnava, Slovakia, 19 al 22 de Septiembre (2010a).

Ayuga-Téllez, E.; González-García, C.; Grande-Ortíz, M.A.; Reyes-Hernández, B. y GarcíaVentura, C., Selection of basic topics for the knowledge of statistics in engineering. Actas del Joint International IGIP-SEFI 2010, Trnava, Slovakia, 19 al 22 de Septiembre (2010b).

BOE, Boletín Oficial del Estado (en línea) 2007. Real Decreto 1393/2007, de 29 de octubre, por el que se establece la ordenación de las enseñanzas universitarias oficiales. http://www.boe.es/boe/dias/2007/10/30/pdfs/A44037-44048.pdf. Acceso: 12 de Noviembre (2009)

Bornmann, L., S. Mittag y H.-D. Daniel, Quality assurance in higher education meta-evaluation of multi-stage evaluation procedures in Germany, Higher Education: 52(4) 687-709 (2006).

Cardona, D. M. y J. M. Sánchez. Indicadores Básicos para Evaluar el Proceso de Aprendizaje en Estudiantes de Educación a Distancia en Ambiente e-learning. Formación Universitaria: 3(6), 1532 (2010).

Cancela, A.; Sánchez, A.; Gandón, R. y M. J. Rey. La Gestión de Calidad ante la Actual Dimensión Universitaria en España. Formación Universitaria: 3(2), 29-36 (2010).

Childress, M. Data-Driven Decision Making: The Development and Validation of an Instrument to Measure Principals' Practices, Academic Leadership Journal, ISSN: 1533-7812 (en línea), 9(2) 2011. http://www.academicleadership.org/article/data-driven-decision-making-the-developmentand-validation-of-an-instrument-to-measure-principals-practices . Acceso: 17 de Enero (2012).

delMas, R.; J. Garfield, A. Ooms, y B. Chance. Assessing Students' Conceptual Understanding After a First Course in Statistics, Statistics Education Research Journal: 6(2), 28-58 (2007).

Damián J. S., E. Montes Pauda y L.J. Arellano Mont. Los Estudios de Opinión de Empleadores. Estrategia para Elevar la Calidad de la Educación Superior no Universitaria. Revista Iberoamericana sobre Calidad, Eficacia y Cambio en Educación: 8(3), 180-203 (2010).

Díaz, C. y C. Batanero, University students' knowledge and biases in conditional probability reasoning. International Electronic Journal of Mathematics Education, ISSN: 1306-3030 (en linea), 4(3), 131-162, 2009. http://www.iejme.com/ /032009/full.pdf. Acceso 16 de Enero (2011).

Downs, C.T. What should make up a final mark for a course? An investigation into the academic performance of first year Bioscience students. Assessment \& Evaluation in Higher Education: 31(3), 345-364 (2006).

Dym, C.L., A.M., Agogino, O. Eris, D.D. Frey y L.J. Leifer, Engineering Design Thinking, Teaching, and Learning. Journal of Engineering Education: 94(1), 103-120 (2005). 
García Martínez, V., S.P. Aquino Zúñiga, A. Guzmán Sala y A. Medina Meléndez, Propuesta Para El Desarrollo de Instrumentos de Autoevaluación para Programas Educativos a Distancia, Revista Electrónica Actualidades Investigativas en Educación, ISSN 1409-4703 (en línea), 11(2) 2011, http://revista.inie.ucr.ac.cr/ediciones/controlador/Article/accion/show/articulo/propuesta-para-eldesarrollo-de-instrumentos-de-autoevaluacion-para-programas-educativos-a-distancia.html. Acceso: 18 de Enero (2012).

Gardner, P. L. y I. Hudson, University Students' Ability to Apply Statistical Procedures, Journal of Statistics Education (en linea), 7(1), 1999.

http://www.amstat.org/publications/jse/secure/v7n1/gardner.cfm. Acceso: 19 de Enero (2012).

Garfield, J. B., Assessing statistical reasoning. Statistics Education Research Journal: 2(1), 22-38 (2003).

Garfield, J. y A. Ahlgren, Difficulties in Learning Basic Concepts in Probability and Statistics: Implications for Research, Journal for Research in Mathematics Education: 19(1), 44-63 (1988).

GNE, Group of National Experts on the AHELO Feasibility Study, Progress Report On The Engineering Strand, $6^{\circ}$ meeting of the AHELO GNE, Paris, Francia, 28-29 de Marzo (2011)

Kessell, J.K.; G.J., Wingenbach y D.L. Lawver, Relationships Between Special Education Confidence, Knowledge, And Selected Demographics For Agricultural Education Student Teachers. Journal of Agricultural Education: 50(2) 52-61 (2009).

Koehn, E. ABET Program Criteria: Review and Assessment for a Civil Engineering Program. Journal of Engineering Education, ASEE: 90(3), 445-455 (2001).

Maher, A. Learning Outcomes in Higher Education: Implications for Curriculum Design and Student Learning. Journal of Hospitality, Leisure, Sport and Tourism Education: 3(2), 46-54 (2004).

Martínez-Falero, E. y S. González-Alonso. Quantitative Techniques in Landscape Planning, CRC Lewis Publishers, 204-235, Boca Ratón, Florida, USA (1995).

Martínez-Padilla, J.H. y J.A. Pérez-González. Efecto de la Trayectoria Académica en el Desempeño de Estudiantes de Ingeniería en Evaluaciones Nacionales. Formación Universitaria: 1(1), 3-12 (2008)

Nagy, G. y B. Sikdar. Classification and Evaluation of Examples for Teaching Probability to Electrical Engineering Students. IEEE Transactions on Education: 51(4), 476-483 (2008).

Nowatzki, E.A. Model for education, professional preparation, and licensure of civil engineers. Journal of Professional Issues in Engineering Education and Practice: 130(4), 269-279 (2004).

OECD, Organisation for Economic Cooperation and Development, Testing student and university performance globally: OECD's AHELO (en linea) 2009.

http://www.oecd.org/document/22/0,3746,en_2649_35961291_40624662_1_1_1_1,00.html.

Acceso: 17 de Enero (2012)

Oloruntegbe, K.O., S.N.A.S. Zamri, R.M. Saat y G.M. Alam, Development and validation of measuring instruments of contextualization of science among malaysian and nigerian serving and preservice chemistry teachers. International Journal of the Physical Sciences: 5(13), 2075-2083 (2010).

Parmenter K y J. Wardle, Evaluation and design of nutrition knowledge measures. J Nutr Educ: 32, 269-277 (2000) 
Peña, D., A. Prat y R. Romero. La Enseñanza de la Estadística en las Escuelas Técnicas. Estadística Española: 32(123), 147-200 (1990).

Praslova, L. Adaptation of Kirkpatrick's four level model of training criteria to assessment of learning outcomes and program evaluation in Higher Education. Educational Assessment Evaluation and Accountability: 22 (3), 215-225 (2010).

Quiroga Parra, D., Metodología para hacer prospectiva empresarial en la sociedad de la información y el conocimiento. Revista Economía y Administración: 70 (70), 25- 44 (2008)

Schwenk, J., F. Hossain y D.A. Huddleston, Computer-Aided Visualization Tool for Stochastic Theory Education in Water Resources Engineering, Computer Applications in Engineering Education: 17(4), 398-411 (2009).

Shaughnessy, J. M., Research in Probability and Statistics: Reflections and Directions. In Handbook of Research on Mathematics Teaching and Learning, ed. D. A. Grouws, MacMillan, pp. 465-494, New York, USA (1992).

Shizuka, T., O. Takeuchi, T. Yashima, y K. Yoshizawa, A comparison of three- and four-option English tests for university entrance selection purposes in Japan. Language Testing: 23(1), 35-57 (2006)

van de Watering, G., D. Gijbels, F. Dochy y J. van der Rijt, Students' assessment preferences, perceptions of assessment and their relationships to study results. Higher Education: 56(6), 645658 (2008).

Vendramini, C.M.M., S.O. Nogueira y F. L. Lopes. The statistics items in the Brazilian National Student Performance Exam (ENADE) Actas del the 8th International Conference on Teaching Statistics. Ljubljan, Eslovenia, 4 al 6 de Julio (2010).

Venter, I. Construction of a valid and reliable test to determine knowledge on dietary fat of highereducated young adults. South African Journal of Clinical Nutrition: 21(3), 133-139 (2008). 